\title{
Effect of different calcination environments on the vanadium phosphate catalysts for selective oxidation of propane and n-butane
}

\begin{abstract}
Vanadium phosphate catalysts were synthesized via VOPO42H2O and were calcined in two different hydrocarbon reaction environments, i.e.n-butane/air and propane/air. Both catalysts are denoted VPDB and VPDP, respectively. Both catalysts exhibited a good crystalline with characteristic peaks of pyrophosphate phase. However, the peaks for VPDP are shown to be more prominent than those of VPDB. BET surface area showed that VPDB gave higher surface area $(23 \mathrm{~m} 2 \mathrm{~g} 1)$ compared to VPDP $(18 \mathrm{~m} 2 \mathrm{~g} 1)$. The average V valence state for VPDP is 4.08 and the higher V valence state for VPDB is 4.26 due to higher amount of VV for VPDB. Furthermore $14.2 \%$ of VIII was found for VPDP but none for VPDB. SEM micrographs clearly revealed that the morphologies of both catalysts composed of plate-like crystallite that was arranged into the characteristic of rosette cluster. However, the catalyst calcined in n-butane/air environment (VPDB) resulted in an increment of the amount of plate-like crystal formed in the rosette rosebud agglomerates. TPR in H2 profiles of both catalysts gave two reduction peaks corresponding to two kinetically different oxygen species which were associated with VVand VIV phases, respectively. VPDB removed larger amount of active oxygen species linked to VIV phase which eventually caused a higher conversion rate in the selective oxidation of n-butane and propane to maleic anhydride and acrylic acid, respectively.
\end{abstract}

Keyword: VPO, n-Butane oxidation,Propane oxidation, Maleic anhydride, Acrylic acid 